\title{
Anxiety and vestibular disorders: a frequent related
}

\section{Short communication}

Anxiety disorders are a group of associated conditions which have one thing in common: persistent, excessive fear or worry in situations that are not intimidating. They are the most frequent mental health concern. There are several types of anxiety disorders but the one thing they have in common is their impact on daily activities. Anxiety can influence the concentrate ability, ${ }^{1}$ sleep and carry out usual labor, home or school tasks. Subjects with anxiety disorders often experience obliged to avoid stressful situations and in extreme cases keep away from going out overall. Corporal symptoms are regular and include shortness of breath, heart increase pulsation and tremulous hands. Women have more probability to develop anxiety than men, but the reason is not understandable.

Dizziness is a common complaint in the primary care scenery and is one of the most common reasons for consultation to specialist care. It usually happen associated to anxiety disorders. If one is experiencing anxiety, dizziness can result. On the other hand, dizziness can be anxiety-producing. The corporal balance works at a subconscious ${ }^{2}$ level. It provide many functions related to oculomotor control, balance regulation, and perception of self-motion; with extensive cortical connections; and is multimodal, integrating vestibular, proprioceptive, and visual systems. The vestibular system is located in the inner ear. Present studies about anxiety and vestibular function indicate that there may be a relationship and vestibular disorders can lead to a wide range of symptoms (from simple, such as vertigo and visual and balance issues, to emotional, memory, and self-perception alterations). ${ }^{3}$

For the reason that vestibular deficits are risk issue for the progress of secondary psychiatric disorders, a somatopsychic theory has been suggested. In opposition, subjects with psychiatric disorders often report dizziness as a concomitant phenomenon. This connection of vestibular and psychiatric symptoms in patients with dizziness is a constant debate. Additionally, psychological factors, such as anxiety and depressive disorders, might influence clinical arrangements and therapeutic results for balance complaints. Nevertheless, previous researches have alert only on the points of co-morbidity between dizziness and psychiatric distress. On this way, a large percentage of patients with vestibular vertigo developed secondary psychiatric disorders over the course of their disease. The association mechanism between anxiety and symptoms of dizziness remains unclear, although a number of studies have suggested that it is related to the considerable overlap between the neuroanatomical regions and neurotransmitters concerned to the vestibular system and the pathways implicated in emotional conditions. ${ }^{4}$

The dizziness that comes with anxiety is often expressed as a sense of light-headedness or wooziness. There may well be a sensation of motion or spinning inside rather than in the environment. Sometimes there is a sense of swaying even though you are standing still. Settings as grocery stores, crowded malls or wide open spaces, for example, can cause a sense of disequilibrium. ${ }^{5}$ These symptoms are caused by legitimate physiologic changes within the brain. Like other brain diseases, anxiety disorders could be caused by problems in the
Volume I Issue 2 - 2017

\author{
Lilian Felipe \\ Speech \& Hearing Sciences, Lamar University, USA
}

Correspondence: Lilian Felipe, Speech \& Hearing Sciences, Lamar University, USA, Email lilianfelipe@id.uff.br

Received: August 20, 2017 | Published: October 16, 2017

functioning of brain circuits that regulate fear and other emotions. Several researches have shown that severe stress can change the pathway nerve cells in these circuits transmit information from one area to another. Other studies have revealed anxiety disorders have changes in some brain structures that control memories linked with strong emotions. Anxiety seems to be a particular problem in patients with acute vestibular disorders. In patients with chronic dizziness, anxiety is present but less pronounced. Frequently the anxiety and the dizziness must be treated together in order for improvement to be made. A comprehensive multidisciplinary assessment of dizziness promises an effective diagnosing and treatment dizziness. ${ }^{6}$

Scales must be used to screen for anxiety/depression in these patients to assist in the early diagnosis and treatment, and psychologists/psychiatrist should be considered to help with the diagnosis and treatment, if necessary. Cognitive-behavioral therapy and rehabilitation of vestibular function or drug therapy are also necessary for the improvement of quality of life. Besides treating the dizziness, the anxiety possibly will require medical treatment. Mainly management engages psychological therapy, cognitive-behavioral rehabilitation and drug prescription. Systematic investigations of the treatment of patients with vestibular symptoms and anxiety are lacking, despite the fact that prevalence, associated costs, and disabilities recommend that they are required. An interdisciplinary treatment is more than recommended in all cases. ${ }^{7}$

\section{Acknowledgments}

None.

\section{Conflict of interest}

The author declares no conflict of interest.

\section{References}

1. Best C, Eckhardt Henn A, Tschan R, et al. Psychiatric morbidity and comorbidity in different vestibular vertigo syndromes. Results of a prospective longitudinal study over one year. J Neurol. 2009;256(1):5865.

2. Eckhardt Henn A, Best C, Bense S, et al. Psychiatric comorbidity in different organic vertigo syndromes. J Neurol. 2008;255(3):420-428.

3. Frommberger U, Hurth Schmidt S, Dieringer H, et al. Panic disorder and vertigo. On the psychopathologic differentiation between neurologic and psychiatric disease. Nervenarzt. 1993;64(6):377-383. 
4. Holmberg J, Karlberg M, Harlacher U, et al. Treatment of phobic postural vertigo. A controlled study of cognitive-behavioral therapy and self-controlled desensitization. J Neurol. 2006;253(4):500-506.

5. Lahmann C, Henningsen P, Brandt T, et al. Psychiatric comorbidity and psychosocial impairment among patients with vertigo and dizziness. $J$ Neurol Neurosurg Psychiatry. 2015;86(3):302-328.
6. Strupp M, Dieterich M, Zwergal A, et al. Peripheral, central and functional vertigo syndromes. Nervenarzt. 2015;86(12):1573-1584.

7. Tschan R, Best C, Wiltink J, et al. Persistence of symptoms in primary somatoform vertigo and dizziness: a disorder lost in health care? J Nerv Ment Dis. 2013;201(4):328-333. 